\title{
Constraints on terrestrial gamma ray flash production from satellite observation
}

\author{
B. E. Carlson, ${ }^{1}$ N. G. Lehtinen, ${ }^{1}$ and U. S. Inan ${ }^{1}$ \\ Received 9 January 2007; revised 26 February 2007; accepted 22 March 2007; published 25 April 2007.
}

[1] Terrestrial gamma-ray flash (TGF) production has been modeled by several emission mechanisms. In spite of extensive studies of these mechanisms and the existing satellite data, significant uncertainties exist about the nature of the source. In this paper, we present a method to develop approximate production-mechanism-independent constraints on TGF production on the basis of Monte Carlo simulation of gamma-ray propagation through the atmosphere. A broad range of possible source parameters are simulated, based on assumptions of a simplified source with varying general properties. The results of the simulations are compared with averaged satellite data, from which constraints are derived. Constraints on the basis of photon fluence and spectral shape are presented and indicate a source of $10^{3}-10^{5} \mathrm{~J}$ at an altitude of $15-20 \mathrm{~km}$. Possible constraints based on the lateral distance between the location of TGF production and the spacecraft nadir point is also discussed. Citation: Carlson, B. E., N. G. Lehtinen, and U. S. Inan (2007), Constraints on terrestrial gamma ray flash production from satellite observation, Geophys. Res. Lett., 34, L08809, doi:10.1029/2006GL029229.

\section{Introduction}

[2] Terrestrial gamma-ray flashes (TGFs) have attracted a great deal of attention since their discovery as a few brief ( $\sim 1 \mathrm{~ms}$ ), upward-directed flashes of gamma rays by the Burst And Transient Source Experiment (BATSE) on board the Compton Gamma-ray Observatory satellite [Fishman et al., 1994]. Recently, the Reuven Ramaty High Energy Solar Spectroscopic Imager (RHESSI) satellite has detected many more events, measuring an average spectrum extending up to $20 \mathrm{MeV}$ [Smith et al., 2005].

[3] Various emission mechanisms have been proposed to explain these brief energetic bursts of radiation, typically accounting for the gamma rays by bremsstrahlung from runaway relativistic electron (RRE) beams. Such mechanisms include quasi-electrostatic (QES) acceleration above thunderclouds immediately after lightning discharge [Lehtinen et al., 1999; Babich et al., 2004] and acceleration by lightning-induced electromagnetic pulses (EMPs) [Milikh and Valdivia, 1999; Inan and Lehtinen, 2005]. QES models typically produce upward-directed gammarays from a range of altitudes above the thundercloud and require a large charge moment change to produce the necessary $E$-field. EMP models tend to produce emissions

\footnotetext{
${ }^{1}$ Space, Telecommunications, and Radioscience Laboratory, Stanford University, Stanford, California, USA.
}

Copyright 2007 by the American Geophysical Union. 0094-8276/07/2006GL029229\$05.00 from higher altitudes, and require instead a rapidly-moving current pulse to generate the required EM wave.

[4] Previous experimental results leave the source parameters uncertain. The BATSE low-energy spectral information seems to indicate a source altitude above $30 \mathrm{~km}$ in order to avoid attenuation of the detected low-energy photons [Fishman et al., 1994], while the very hard highenergy spectra observed by RHESSI require such attenuation to account for the hardness observed[Smith et al., 2005; Dwyer and Smith, 2005], and thus predict lower source altitudes. The BATSE data also includes directional information which indicates TGF detections at large nadir angles, suggesting isotropic emission, though the accuracy of BATSE direction finding for such low-fluence events may be questionable according to J. Fishman (personal communication, 2006). Correlations between RHESSI data and very-low-frequency radio (VLF) lightning observations, however, indicates clustering with much smaller nadir angles, suggesting a narrower emission directional distribution [Cummer et al., 2005; Stanley et al., 2006]. Further, many RHESSI and BATSE TGFs may have no correlated lightning [Inan et al., 2006], leading to the possibility of conjugate hemisphere events, or even a generation mechanism that does not necessarily involve lightning discharge.

[5] These various models and conflicting results make it desirable to set constraints on the source of TGF photons. Dwyer and Smith [2005] used an assumed QES electric field to drive a runaway breakdown simulation to examine TGF spectral shape and thus constrain source altitude. In this paper, we focus instead on the known physics of photon propagation to avoid invoking any particular production mechanism. We vary the properties of an assumed photon source over reasonable, production-mechanism-independent ranges, simulate the propagation of the emitted photons, predict resulting satellite observations, and compare the results to existing satellite data to constrain the initial source properties.

\section{Methods}

\subsection{Assumptions and Initial Conditions}

[6] Sacrificing the realistic complexity of simulations of all TGF source mechanisms in favor of simplicity and generality, we consider point-source photon emission with a typical spectrum and specified angular distribution. This allows us to consider wide ranges in possible emission parameters without focusing too much on any hypothetical source mechanism.

[7] Two initial photon spectra relevant to TGF production are considered here. First, the hardest allowable spectrum, $d N / d \mathcal{E}=1 / \mathcal{E}$, is used (hereinafter referred to as $1 / \mathcal{E}$ initial case), with appropriate low- and high-energy limits $(10 \mathrm{keV}$ 


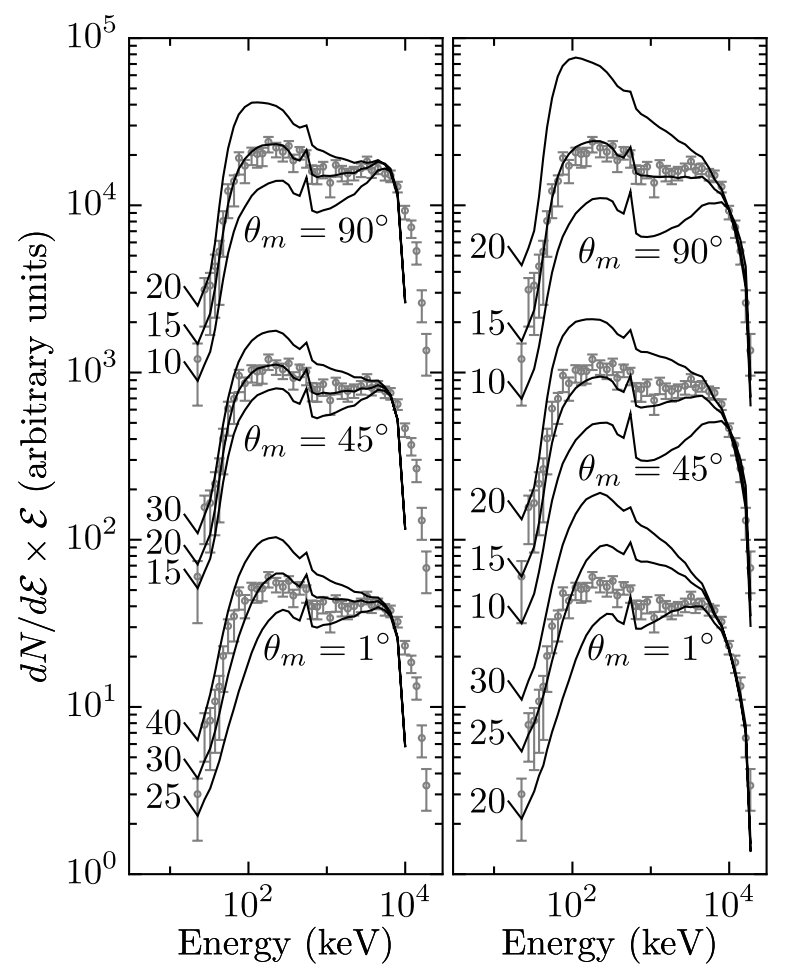

Figure 1. Observed RHESSI average spectra (bars) plotted against MC output spectra convolved with RHESSI response matrix (solid lines) for a variety of initial altitudes (marked in $\mathrm{km}$ at left), normalized to agree at $10 \mathrm{MeV}$. (left) $1 / \mathcal{E}$ initial spectrum, (right) QES initial spectrum.

and $10 \mathrm{MeV}$, mean photon energy $\overline{\mathcal{E}} \approx 1.4 \mathrm{MeV}$ ). The second spectrum used is a softer spectrum of thin-target bremsstrahlung in air emitted by electrons with energies distributed according to a QES simulation with electric field strength $E / E_{t}=2$, where $E_{t}$ is the RRE breakdown threshold, taken from Lehtinen et al. [1999] (hereinafter referred to as QES initial case). Bremsstrahlung here is generated by the GEANT4 package [Agostinelli et al., 2003], and is cut off below $10 \mathrm{keV}$ (mean $\overline{\mathcal{E}} \approx 1.2 \mathrm{MeV}$ ). Though the QES case relies on a particular production mechanism, similar electric fields should produce similar effects, so this spectrum could be taken as representative of such models. This spectrum also allows comparison of our results to existing literature, for example Dwyer and Smith [2005].

[8] The input is constructed as a point source whose altitude is chosen to fall between 10 and $70 \mathrm{~km}$, as sources above $70 \mathrm{~km}$ lack sufficient atmosphere, while photons from sources below $10 \mathrm{~km}$ are heavily attenuated. The source is taken to be a uniform beam diverging with half-angle $\theta_{m}$, ranging from narrow beam to half-isotropic, $1^{\circ}<\theta_{m}<90^{\circ}$, covering the range of interest from the unlikely case of beaming due to bremsstrahlung from unidirectional $35 \mathrm{MeV}$ electrons to wide-angle emissions from more complicated emission mechanisms. The resulting photons are then passed to a Monte Carlo photon transport simulation.

\subsection{Monte Carlo Photon Transport}

[9] The Monte Carlo (MC) photon transport code accounts for all relevant photon interactions. Compton scattering and photoelectric absorption are included as described by Inan et al. [1999]. Free electron production and propagation are ignored, as bremsstrahlung by Compton or photoelectrons is negligible. Pair production and subsequent positron annihilation are included approximately by replacing the primary photon with a pair of $511 \mathrm{keV}$ photons with isotropically chosen opposite momenta (thereby ignoring positron propagation). The Monte Carlo code used here has been compared to GEANT4 [Agostinelli et al., 2003] simulations of identical source and atmospheric conditions spanning the range relevant to our simulations and is found to agree well, deviating by $\leq 15 \%$ for the relevant parameter ranges, while running $\sim 100 \times$ faster, allowing large searches in parameter space as required by this method.

[10] Enough initial photons are simulated to ensure that at least $5 \times 10^{5}$ photons reach satellite altitude (here $600 \mathrm{~km}$ ), requiring between $6 \times 10^{5}$ photons for high altitude sources to $3 \times 10^{9}$ photons for low altitude sources. These photons are then used to simulate satellite data.

\subsection{Satellite Observation}

[11] The resulting photons are binned by location in the satellite orbital plane and convolved with the satellite response matrix to predict a satellite-observed spectrum for each location. Satellite response matrices were obtained for RHESSI from D. Smith (personal communication, 2006) and for BATSE from the Compton Observatory Science Support Center (COSSC) data archive (available at http:// cossc.gsfc.nasa.gov, discsc_drm files) and are averaged over direction.

[12] For a given set of source parameters, comparing this set of predicted spectra to the set of observed TGFs, we can, by assuming no variability in source parameters, identify the most-intense predicted spectrum with the most-intense observed TGF, and therefore the least-intense observed TGF with the minimum intensity threshold relevant to the set of predicted spectra. This is valid if the variation among the sources of the observed TGFs is small enough to not significantly vary the measurable quantities. This is the method used by Dwyer and Smith [2005]. The most- and least-intense RHESSI (and BATSE) TGFs differ by $\sim 5 \times$ in intensity, so all predicted spectra with intensities less than $1 / 5$ th that of the most intense predicted spectrum are dropped from the analysis. This removes the complexity of detection thresholds, noise rates, etc. The result is a set of above-threshold predicted spectra, one for each horizontal location in the satellite plane.

\section{Results and Comparison with Existing Data 3.1. Spectrum Shape}

[13] The predicted average spectrum is calculated by averaging the set of above-threshold predicted spectra over location, giving a predicted average spectrum for each initial altitude, spectrum, and angular distribution. This can easily be compared to experimental data to examine, as in the work of Dwyer and Smith [2005], the question of source altitude. This is shown for the RHESSI and BATSE satellites in Figures 1 and 2. The experimental RHESSI average spectrum data are reproduced from Dwyer and Smith [2005], while the BATSE data shown are taken from time-tagged-event information from the COSSC data archive, background-subtracted, and averaged. The RHESSI 


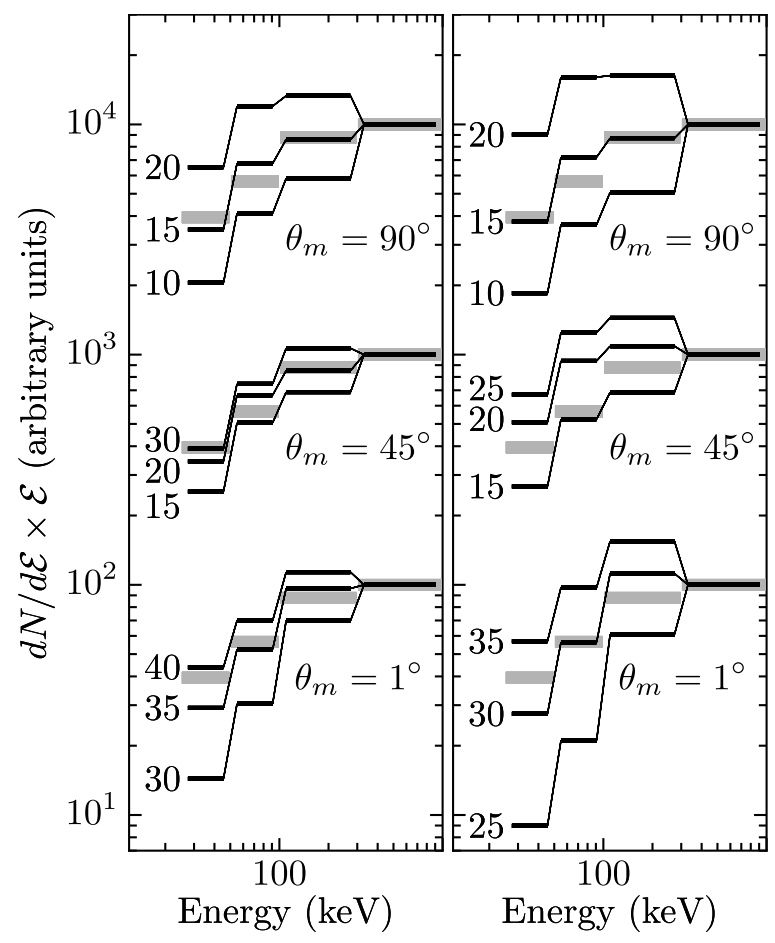

Figure 2. Observed BATSE average spectral data (thick grey lines) plotted against MC output spectrum convolved with BATSE response matrix (black lines) for a variety of initial altitudes (marked in $\mathrm{km}$ at left). (left) $1 / \mathcal{E}$ initial spectrum, (right) QES initial spectrum.

spectra have been renormalized to be equal near $10 \mathrm{MeV}$, following Dwyer and Smith [2005], while the BATSE spectra are renormalized to agree in the $>300 \mathrm{keV}$ bin. For both RHESSI and BATSE, both the $1 / \mathcal{E}$ and QES initial spectra fit quite well for initial angular distributions with $\theta_{m} \geq 45^{\circ}$. The $\theta_{m}=1^{\circ}$ case does not fit as well, likely due to scattering of photons to locations far from the intense central beam and therefore outside the set of abovethreshold locations. This indicates that photons scattered by large angles must be included to explain the observed spectra, suggesting emissions in directions away from the satellite as well as toward, inconsistent with narrow-beam emissions.

[14] The $1 / \mathcal{E}$ case clearly deviates at high energies in the RHESSI spectra, due to the sharp maximum-energy cutoff chosen. Higher cutoffs (data not shown) result in predicted spectra with an excess of high-energy photons, indicating a gradual cutoff in initial photon spectrum starting at $\sim 10 \mathrm{MeV}$. There are potentially other systematic deviations, but the match between our results and the data is already quite good. Therefore, assuming a source spectrum similar to our initial spectra for emissions with half-angle $\theta_{m} \geq 45^{\circ}$, these simulations indicate a source altitude in the $15-20 \mathrm{~km}$ range, consistent with Dwyer and Smith [2005].

[15] Simulations with $1 / \mathcal{E}$ initial spectra result in similar or slightly higher best-fit initial altitudes than the QES initial conditions. This is expected, as the softer QES initial spectrum requires greater attenuation of low-energy photons to match the data, implying some constraint relating best-fit initial altitude and initial spectrum. However, the QES and $1 / \mathcal{E}$ initial spectra result in similar altitudes, so this difference is relatively unimportant.

\subsection{Maximum TGF Intensity}

[16] The maximum number of photons observable by a satellite for a given set of source parameters can be estimated by integrating the most-intense predicted spectrum. This is easily compared to the most-intense observed TGF for the given satellite to allow analysis of emission energy. The points in source parameter space where the predicted maximum number of photons observable by a satellite matches the most- and least- intense observations are shown as shaded and dashed in Figure 3, indicating the minimum TGF energy necessary ( $\mathrm{max} / \mathrm{min}$ : RHESSI 101/17 from Smith et al. [2005], BATSE 1000/100 from the COSSC data archive). The allowed regions for RHESSI and BATSE are not necessarily coincident, as the satellites have different response matrices. Only one input spectrum is shown here $(1 / \mathcal{E})$ as the results for the two input spectra differ by a factor $\leq 2$, small compared to the factor of $10^{3}$ difference between the $\theta_{m}=1^{\circ}$ and $\theta_{m}=90^{\circ}$ cases.

\subsection{Horizontal Extent}

[17] The horizontal extent of TGF emissions in the satellite orbital plane is also of interest as it is related to the probability of detection and can be compared with BATSE data or RHESSI/VLF correlation. Here we measure this horizontal extent by calculating the standard deviation of the Cartesian $y$-coordinate $\left(\sigma_{y}\right)$ of the locations associated with the set of above-threshold predicted spectra. The use of a single coordinate allows separation of the directions in the satellite plane perpendicular and parallel to an off-zenith emission direction, such that the possibility of beaming along the geomagnetic field can be examined. Under the threshold scheme used here, $\sigma_{y}$ is only a function of source

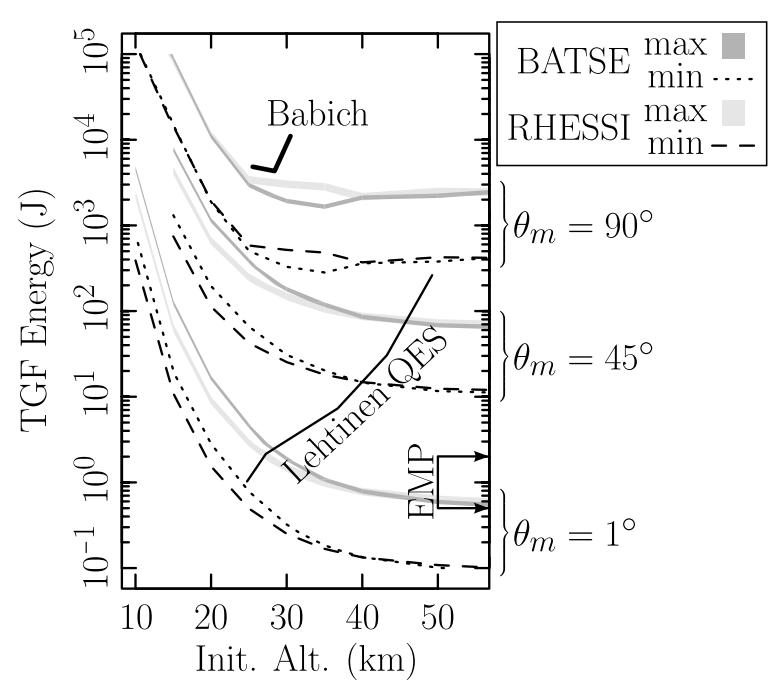

Figure 3. TGF energy vs. source altitude, points for which most-intense predicted observation matches RHESSI and BATSE most- and least- intense TGF ( $\mathrm{max} / \mathrm{min}$ ) shown as shaded and dashed as indicated for initial angular distributions marked by $\theta_{m}$. Models as marked: "EMP" [Inan and Lehtinen, 2005]; "Lehtinen QES" [Lehtinen et al., 1999]; "Babich" [Babich et al., 2004]. 


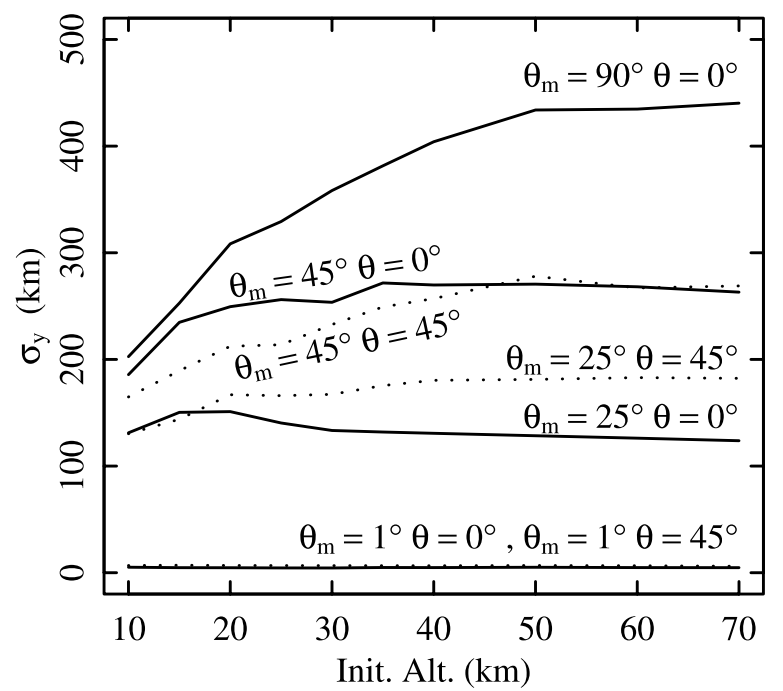

Figure 4. Horizontal extent of TGF emissions in the satellite orbital plane ( $\sigma_{y}$ as described in text) vs. source altitude. Labels indicate half-angle of initial angular distribution $\left(\theta_{m}\right)$ followed by the angle from zenith of the center of the emission (solid lines, zenith-directed emission; dotted lines, $45^{\circ}$ from zenith).

altitude and initial angular distribution, see Figure 4. Results for high-altitude sources accurately reflect the emission mechanism, while emissions from low-altitude sources lose this information by scattering. Low-altitude, large $\theta_{m}$ emissions are focused by the greater attenuation of large-zenithangle photons, while smaller- $\theta_{m}$ emissions do not show this effect as much. The $\theta_{m}=1^{\circ}$ beam produces a very small lateral spread regardless of altitude due to the dominance of unscattered photons in the threshold method used here.

[18] Beaming of emissions along geomagnetic field lines due to gyration of the avalanche electrons along the geomagnetic field (relevant for high-altitude avalanches) should produce a systematic offset between source location and the subsatellite point (subsatellite point systematically closer to the geomagnetic equator than the source). The horizontal extent in directions perpendicular to the deviation from vertical is not drastically affected (consider the dashed curves for emissions $45^{\circ}$ from vertical, Figure 4), so this measure may still be useful.

[19] Recent measurements of lightning-produced radio atmospherics correlated to RHESSI TGFs show atmospheric-subsatellite clustering on a $130 \mathrm{~km}$ to $300 \mathrm{~km}$ scale with no noticed offset indicative of beaming along the geomagnetic field [Cummer et al., 2005; Stanley et al., 2006]. A horizontal extent of $\geq 130 \mathrm{~km}$ is inconsistent with a simple narrow beam, but low-altitude sources with wider angular emissions tend to have similar spread due to scattering. Further searches are needed to refine this constraint, and may add the possibility of spectral or intensity constraints involving distance or angle.

\section{Discussion}

[20] The above constraints establish relations between TGF source parameters and satellite measurements. For example, consider an isotropic emission mechanism. The observed spectra and intensity then require a source altitude of $15-20 \mathrm{~km}$ with a mechanism capable of producing $10^{4}-10^{5} \mathrm{~J}$ of total gamma-ray energy. We then predict a TGF sourcesubsatellite distance spread of $\sim 300 \mathrm{~km}$, which may or may not match further TGF-lightning correlation searches.

[21] Taking our assumptions as valid and using mismatch with experimental data to rule out possible source parameters, our constraints suggest gamma-ray emissions of $10^{3}-$ $10^{5} \mathrm{~J}$, with a QES-style spectrum emitted with an angular spread of $\theta_{m} \geq 45^{\circ}$ from $15-20 \mathrm{~km}$ altitude.

[22] However, the methods used here are only valid up to the assumptions involved (point-source emission, simple spectrum, etc). Any real emission mechanism will partially violate these assumptions, so the results should thus be taken as rough guidelines. QES models, for example, predict emission regions many kilometers high. However, point-source emissions from altitudes in that range should yield similar results. Model-specific calculations are necessary to determine if a truly consistent mechanism has been derived, but such calculations should still fit within the rough constraints brought out here.

[23] A comparison of the intensity constraints with several existing production models is included in Figure 3. The QES models due to Lehtinen et al. [1999] (beamed emission) and Babich et al. [2004] (isotropic emission) are shown, together with the EMP model due to Inan and Lehtinen [2005] (geomagnetic field-aligned emission). Each production model is shown for various of sets of parameters, with the total emitted energy and altitude of peak emission for each set shown as a point. These mechanisms can produce the necessary TGF energy with QES-style spectra, but produce emissions from higher altitudes than the spectral constraints in Figures 1 and 2 indicate.

[24] It is hoped that this paper will motivate refinements of existing models of TGF production to better fit the existing data. Further analysis of MC simulation results may also yield new significant constraints, including the possibility of constraining the RRE avalanche electron energy distribution. VLF TGF-lightning correlation searches should allow the addition of horizontal extent constraints on the emission angular distribution and altitude, and may possibly show the effects of beaming along the geomagnetic field. Future data from RHESSI or results from upcoming experiments such as TARANIS will also help additionally constrain TGF parameters. Such further constraints are easily implemented in this framework.

[25] Acknowledgments. This work made use of BATSE data from the HEASARC online service provided by NASA/GSFC and was supported by the Stanford University Benchmark Fellowship program and NSF/CEDAR grant ATM-0535461.

\section{References}

Agostinelli, S., et al. (2003), G4-A simulation toolkit, Nucl. Instrum. Methods Phys. Res., Sect. A, 506(3), 250-303.

Babich, L. P., R. I. Il'kaev, I. M. Kutsyk, A. Y. Kudryavtsev, R. A. RousselDupré, and E. M. Symbalisty (2004), Analysis of atmospheric gamma ray bursts based on the mechanism of generation of relativistic electron avalanches, Geomagn. Aeron., 44(2), 243-251.

Cummer, S. A., Y. Zhai, W. Hu, D. M. Smith, L. I. Lopez, and M. A. Stanley (2005), Measurements and implications of the relationship between lightning and terrestrial gamma ray flashes, Geophys. Res. Lett., 32, L08811, doi:10.1029/2005GL022778.

Dwyer, J. R., and D. M. Smith (2005), A comparison between Monte Carlo simulations of runaway breakdown and terrestrial gamma-ray flash observations, Geophys. Res. Lett., 32, L22804, doi:10.1029/2005GL023848. 
Fishman, G. J., et al. (1994), Discovery of intense gamma-ray flashes of atmospheric origin, Science, 264(5163), 1313-1316.

Inan, U. S., and N. G. Lehtinen (2005), Production of terrestrial gamma-ray flashes by an electromagnetic pulse from a lightning return stroke, Geophys. Res. Lett., 32, L19818, doi:10.1029/2005GL023702.

Inan, U. S., N. G. Lehtinen, S. J. Lev-Tov, M. P. Johnson, T. F. Bell, and K. Hurley (1999), Ionization of the lower ionosphere by $\gamma$-rays from a magnetar: Detection of a low energy $(3-10 \mathrm{keV})$ component, Geophys. Res. Lett., 26(22), 3357-3360.

Inan, U. S., M. Cohen, N. G. Lehtinen, R. Said, D. M. Smith, and L. I. Lopez (2006), Terrestrial gamma ray flashes and lightning discharges, Geophys. Res. Lett., 33, L18802, doi:10.1029/2006GL027085.

Lehtinen, N. G., T. F. Bell, and U. S. Inan (1999), Monte Carlo simulation of runaway $\mathrm{MeV}$ electron breakdown with application to red sprites and terrestrial gamma ray flashes, J. Geophys. Res., 104(A11), $24,699-24,712$
Milikh, G., and J. A. Valdivia (1999), Model of gamma ray flashes due to fractal lightning, Geophys. Res. Lett., 26(4), 525-528.

Smith, D. M., L. I. Lopez, R. P. Lin, and C. P. Barrington-Leigh (2005), Terrestrial gamma flashes observed up to $20 \mathrm{MeV}$, Science, 307(5712), $1085-1088$

Stanley, M. A., X.-M. Shao, D. M. Smith, L. I. Lopez, M. B. Pongratz, J. D. Harlin, M. Stock, and A. Regan (2006), A link between terrestrial gamma-ray flashes and intracloud lightning discharges, Geophys. Res. Lett., 33, L06803, doi:10.1029/2005GL025537.

B. E. Carlson, U. S. Inan, and N. G. Lehtinen, STAR Lab, Stanford University, 350 Serra Mall, Packard Building, Stanford, CA 94305-9515, USA. (brantc@stanford.edu; inan@stanford.edu; nleht@stanford.edu) 